\title{
Non-Alcoholic Fatty Liver Disease Modifies Serum Gamma- Glutamyl Transferase in Cigarette Smokers
}

\author{
Ebenezer T. Onia ${ }^{a}$, , Vincent Figueredo ${ }^{\mathrm{b}}$, Ehimen Aneni ${ }^{\mathrm{c}}$, Emir Veladar ${ }^{\mathrm{d}}$, John W. McEvoy ${ }^{\mathrm{e}}$, \\ Michael J. Blahae, f, Roger S. Blumenthale, Raquel D. Conceicaog, Jose A.M. Carvalhog, \\ Raul D. Santosg, h, Khurram Nasir ${ }^{\mathrm{i}}$
}

\begin{abstract}
Background: Serum gamma-glutamyl transferase (GGT) is a marker of oxidative stress, associated with increased cardiovascular (CV) risk. The impact of smoking on oxidative stress may be aggravated in individuals with non-alcoholic fatty liver disease (NAFLD). We aimed to ascertain the association of smoking on GGT levels in the presence or absence of NAFLD.
\end{abstract}

Methods: We evaluated 6,354 healthy subjects (43 \pm 10 years, $79 \%$ males) without clinical cardiovascular disease (CVD) undergoing an employer-sponsored physical between December 2008 and December 2010. NAFLD was diagnosed by ultrasound and participants were categorized as current or non-smokers by self report. A multivariate linear regression of the cross-sectional association between smoking and GGT was conducted based on NAFLD status.

Results: The prevalence of NAFLD was $36 \%(\mathrm{n}=2,299)$ and 564 $(9 \%)$ were current smokers. Smokers had significantly higher GGT levels in the presence of NAFLD $(\mathrm{P}<0.001)$. After multivariable adjustment, current smoking was associated with $4.65 \mathrm{IU} / \mathrm{L}$ higher

Manuscript submitted December 31, 2019, accepted January 22, 2020 Published online July 22, 2020

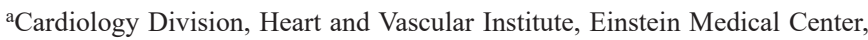
Philadelphia, PA, USA

bSt. Mary Medical Center, 1203 Langhorne-Newtown Road, Suite 320, Langhorne, PA 19047, USA

${ }^{\mathrm{c} S e c t i o n}$ of Cardiovascular Medicine, Department of Medicine, Yale University School of Medicine

${ }^{\mathrm{d} C e n t e r}$ of Advanced Analytics, Baptist Health South Florida, 8900 North Kendall Drive, Miami, FL 33176, USA

${ }^{\mathrm{e}}$ The Johns Hopkins Ciccarone Center for the Prevention of Heart Disease, Baltimore, MD, USA

fDepartment of Epidemiology, Johns Hopkins Bloomberg School of Public Health, Baltimore, MD, USA

gPreventive Medicine Center Hospital Israelita Albert Einstein, Sao Paulo, Brazil ${ }^{h}$ Lipid Clinic-Heart Institute (InCor) University of Sao Paulo Medical School Hospital, Sao Paulo, Brazil

${ }^{i}$ Houston Methodist, Debakey Heart and Vascualr Institute, Houston, TX, USA ${ }^{j}$ Corresponding Author: Ebenezer T. Oni, Cardiology Division, Heart and Vascular Institute, Einstein Medical Center, Philadelphia, PA, USA.

Email: oniebene@einstein.edu

doi: https://doi.org/10.14740/jocmr3932
GGT level, $\mathrm{P}<0.001$, compared to non-smokers. When stratified by NAFLD, the magnitude of this association was higher in subjects with NAFLD ( $\beta$-coefficient: $11.12 ; 95 \%$ confidence interval $(\mathrm{CI})$ : 5.76 - 16.48; $\mathrm{P}<0.001)$; however, no such relationship was observed in those without NAFLD ( $\beta$ : $-0.02 ; 95 \% \mathrm{CI}:-3.59,3.56$; $\mathrm{P}=0.992)$. Overall the interaction of NAFLD and smoking with GGT levels as markers of oxidative stress was statistically significant.

Conclusions: Smoking is independently associated with significantly increased oxidative stress as measured by GGT level. This association demonstrates effect modification by NAFLD status, suggesting that smoking may intensify $\mathrm{CV}$ risk in individuals with NAFLD.

Keywords: Gamma-glutamyl transferase; Oxidative stress; Non-alcoholic fatty liver disease; Smoking; Cardiovascular risk

\section{Introduction}

Oxidative stress plays a crucial role in cardiovascular disease (CVD) mediating the pathway to atherosclerosis and inflammation $[1,2]$. The oxidative stress theory is based on the assumption that the initial phase of atherosclerosis is dependent on low-density lipoprotein (LDL) oxidative modifications [3].

Gamma-glutamyl transferase (GGT) is a biomarker secreted mostly by the liver and found in epithelial cells of humans. It plays an important role in glutathione metabolism, an important component of the anti-oxidative process $[4,5]$. This marker has been implicated in atherosclerosis, diabetes, metabolic syndrome and increased cardiovascular (CV) risk [6-16]. Elevated GGT levels have also been linked with hepatic steatosis (HS) and liver cancer [14, 17]. While non-alcoholic fatty liver disease (NAFLD) is the leading cause of chronic liver disease in the western world, evidence has also shown it as an independent risk factor for CVD [18, 19].

Similarly, it has been established that cigarette smoking is an important risk factor for atherosclerosis and associated with significant vascular disease [20-23]. Smoking enhances the production of reactive oxygen species which promotes oxidative stress and lipid peroxidation [24-27]. While smoking is associated with oxidative stress, its influence on GGT levels in individuals with NAFLD is not clearly defined. Our study examines the effect of the presence of NAFLD on the associa- 
tion between smoking and oxidative stress by GGT levels in an asymptomatic population.

\section{Materials and Methods}

A total of 6,354 asymptomatic men and women, free of CVD, who had an obligatory clinical and laboratory health evaluation from December 2008 to December 2010 at the Preventive Medicine Center of the Albert Einstein Hospital in Sao Paulo, Brazil were evaluated. The examination protocol has been previously described in prior publications [28]. Individuals were provided with questionnaires for self-reported details of their demographics, medical history, quantitative alcohol consumption, smoking status and medication usage during their clinical visits. We excluded 110 individuals with missing information of either smoking status, GGT levels and presence of steatosis. Individuals with an established history of liver disease were also excluded. Individual smoking status was defined as either current smoker or non-smoker. While current smoker was defined as smoking at least one cigarette in the last 1 month, non-smoker was one who did not smoke in the last month. Diabetes mellitus was identified by a previous physician diagnosis or the use of glucose-lowering medication. Hypertension and dyslipidemia were ascertained by a previous history or the use of medications. Blood pressure measurements were made using an aneroid sphygmomanometer using the recommended method from the American Heart Association [29]. The waist circumference was the smallest diameter between the iliac crest and the costal margin using a plastic anthropometric tape.

All blood specimens were collected after an overnight fast. Plasma lipid, glucose, GGT and liver transaminase levels (alanine aminotransferase (ALT) and aspartate aminotransferase (AST)) levels were measured by standardized automated laboratory tests using a Vitros platform (Johnson \& Johnson Clinical Diagnostics, New Brunswick, New Jersey). High-sensitivity C-reactive protein (hs-CRP) levels were determined by immunonephelometry (Dade-Behring $\mathrm{GMbH}$, Mannhein, Germany). All tests were performed at the Central Laboratory of the Albert Einstein Hospital.

NAFLD was diagnosed after at least a 6-h fast using an ACUSON XP-10 device (Mountain View, CA) and was identified by the presence of an ultrasonographic pattern of a bright liver, with evident contrast between hepatic and renal parenchyma and using a standardized algorithm (indicating HS) [30, 31]. Obesity was defined as a body mass index (BMI) of greater than $30 \mathrm{~kg} / \mathrm{m}^{2}$. The metabolic syndrome (MS) was defined using criteria from the American Heart Association/National Heart, Lung, and Blood Institute published scientific statement on the metabolic syndrome [32]. Patients with $\geq 3$ of the following risks were classified as having the MS: truncal obesity $(\geq 102$ $\mathrm{cm}$ (40 inches) for men and $\geq 88 \mathrm{~cm}$ (36 inches) for women), high blood pressure (blood pressure $\geq 130 / 85 \mathrm{~mm} \mathrm{Hg}$ or the use of antihypertensive medications), hyperglycemia (fasting blood glucose $\geq 100 \mathrm{mg} / \mathrm{dL}$ ), low high-density lipoprotein cholesterol (HDL-C) $(\leq 40 \mathrm{mg} / \mathrm{dL}$ for men and $\leq 50 \mathrm{mg} / \mathrm{dL}$ for women) and hypertriglyceridemia ( $\geq 150 \mathrm{mg} / \mathrm{dL})$ [32]. This study did not involve any animal study and appropriate IRB approval was obtained prior to the administration of surveys to the participants.
All data were de-identified prior to analysis and determined not to meet criteria for protected health information (PHI) [28].

Baseline characteristics of individuals with a combination of the presence or absence of NAFLD and smoking status was compared using the analysis of variance test for continuous variables and the Pearson's $\chi^{2}$ test for categorical variables. The non-parametric Kruskal-Wallis test was used to compare the median of variables with skewed distribution (ALT, AST, GGT and hs-CRP). A multivariate linear regression was used to evaluate associations of smoking and GGT levels. The regression analysis was done by stratifying the presence of NAFLD and obesity to ascertain an independent association. For all regression analyses, a hierarchical model approach was used, adjusting first for age and gender and then simultaneously adjusting for other confounding factors (waist circumference, triglycerides, HDL-C, systolic blood pressure (SBP), fasting glucose, LDL-C, CRP, lipid lowering and antihypertensive medications and alcohol use). Alcohol use was quantified by the alcohol use disorders identification test (AUDIT) score [33]. The AUDIT score was developed and validated by the World Health Organization among men and women in different countries. We categorized a total AUDIT score of $\geq 8$ as high alcohol consumption for men and $\geq 4$ for women [34]. A model including an interaction term for HS and smoking was fitted to assess whether smoking modified the association between HS and GGT. All statistical analyses were performed using STATA statistical software, release 12 (College Station, TX).

\section{Results}

The characteristics of the study population were stratified by the combination of smoking status and the presence of NAFLD (Table 1). There were 6,354 subjects analyzed: 3,731 (59\%) non-smokers without NAFLD, 2,060 (32\%) non-smokers who had NAFLD, 324 (5\%) smokers without NAFLD and 239 (4\%) smokers with NAFLD. There were statistically significant differences in demographics, anthropometric and biochemical profiles between the four groups. Individuals with NAFLD were more likely to be men, older and have a higher burden of risk factors (Table 1). However, smoking combined with the presence of NAFLD further worsened this association. Smokers with NAFLD had the highest prevalence of the MS.

Smokers with NAFLD were more likely to have the highest level of GGT levels ( $\mathrm{P}<0.001)$. Individuals who smoked and had NAFLD had the highest median levels compared to individuals who had NAFLD alone, smoked without NAFLD or were non-smokers without NAFLD. Figures 1 and 2 show the effect of smoking on the GGT levels in the presence and absence of NAFLD. Although the presence of NAFLD alone was associated with a high GGT level, it was even higher when the presence of NAFLD is combined with a positive smoking status (Fig. 1). Figure 2 shows similar effect of cigarette smoking on the quartiles of GGT levels when stratified by the presence of NAFLD. While non-smokers without NAFLD have predominantly GGT levels in the lowest quartiles, there is a steady redistribution to the higher quartiles in smokers without NAFLD, non-smokers with NAFLD and smokers with 


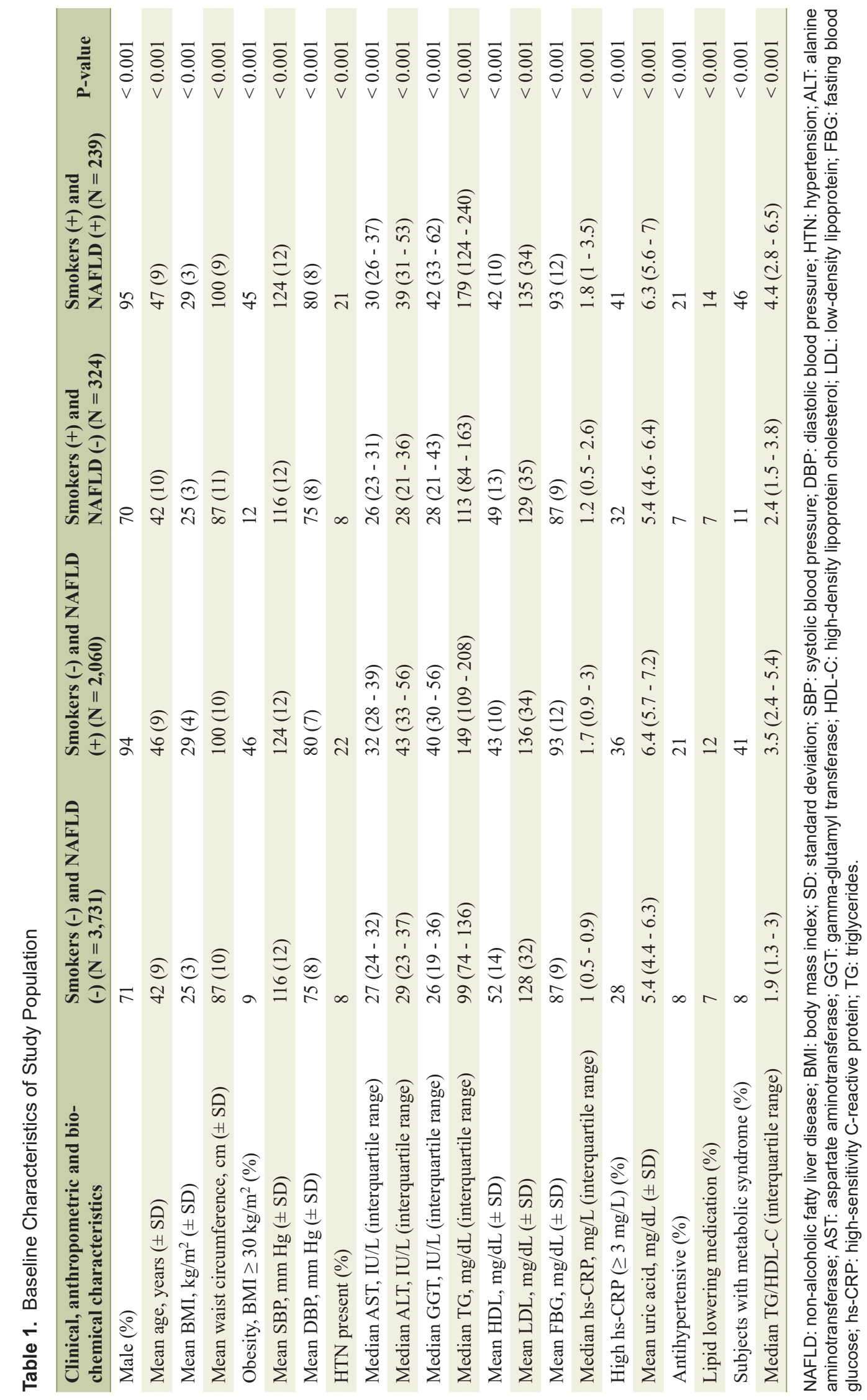




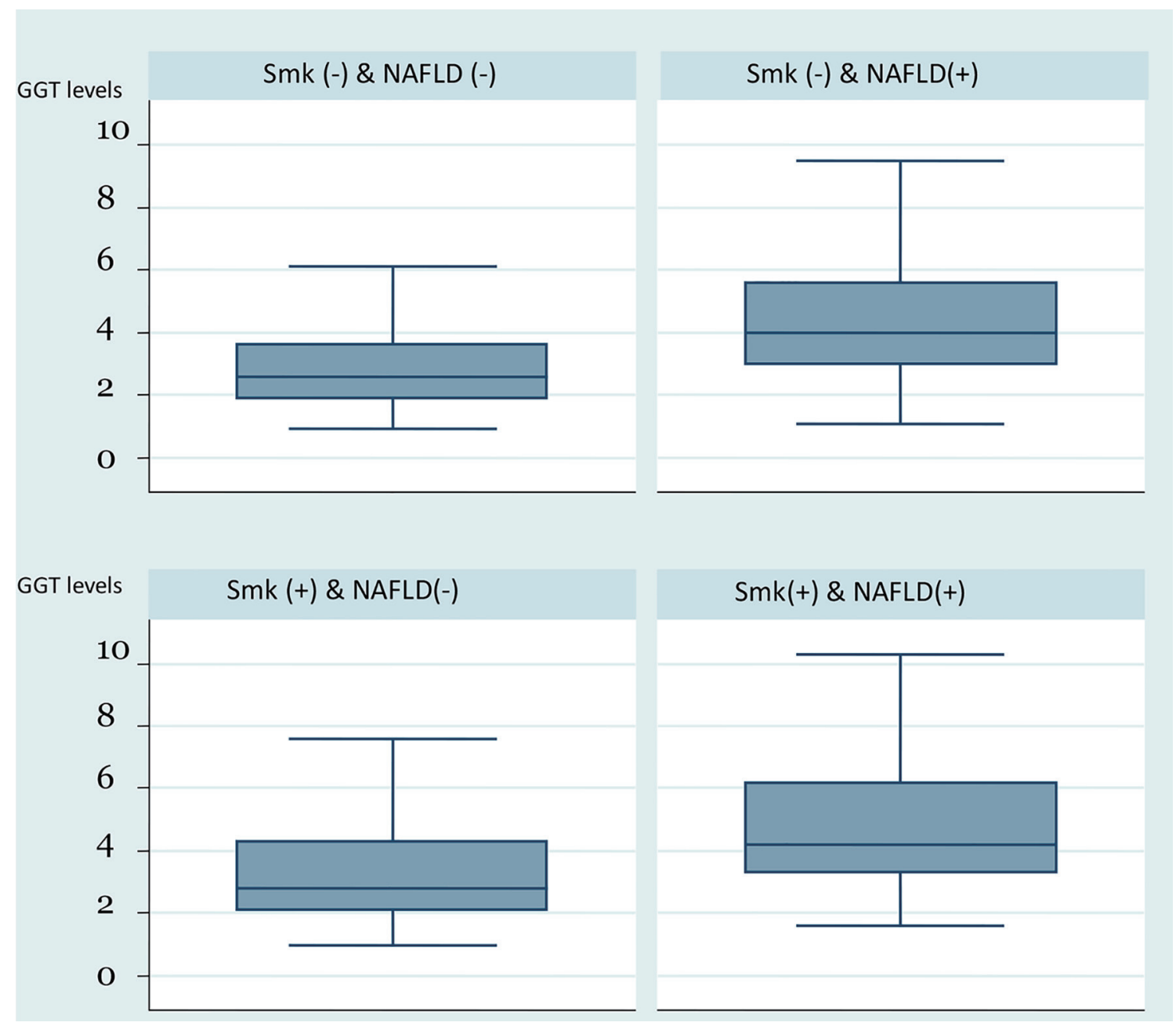

Figure 1. Box plot of GGT levels by the combination of smoking status and presence of HS. GGT: gamma-glutamyl transferase; HS: hepatic steatosis.

NAFLD. The GGT levels in smokers with NAFLD were predominately in the highest quartile. Figure 3 presents a similar relationship when stratified by MS.

Table 2 presents multivariate regression of the relationship between smoking and serum GGT levels. The results are presented from both the unadjusted and adjusted models for possible confounders. The analysis was also stratified by the presence of NAFLD. While there was a significant relationship between smoking and GGT in both the combined population and among individuals with NAFLD, the association was however not significant when NAFLD was absent. In the combined population, the GGT level of smokers was $4.65 \mathrm{IU} / \mathrm{L}$ higher. It was higher among individuals with NAFLD in the stratified analysis, as the GGT level of smokers in this group was 11.12 IU/L higher. The model testing for interaction demonstrated a statistically significant interaction of NAFLD and smoking in the association of smoking on GGT levels. We also found a similar association when stratified by the presence of obesity (Table 3). Obesity interacted with the association of smoking and the GGT levels and we noted the effect of smoking on GGT was independent of gender (Fig. 4).

\section{Discussion}

Our study of 6,354 asymptomatic individuals assessed the impact of smoking on oxidative stress in NAFLD. While $9 \%$ of the population were smokers, $36 \%$ had NAFLD. We demonstrated the presence of NAFLD modified the impact of cigarette smoking on GGT levels. Smokers who had NAFLD had higher GGT levels with evidence of interaction between smoking and NAFLD on the effect of smoking on GGT levels. This effect modification was also demonstrated with obesity and independent of gender.

This findings support prior studies that showed a relationship between smoking, NAFLD and GGT levels [24, 35, 36]. Smoking promotes oxidative stress and has a role in the development and progression of NAFLD [24]. While liver fat is a useful measure of visceral adiposity, it is also associated with increased risk of CVD [19-21].

Cigarette smoking has enormous consequences on health. It has been implicated in the development of NAFLD and known to cause inflammation and oxidative stress. It affects in- 

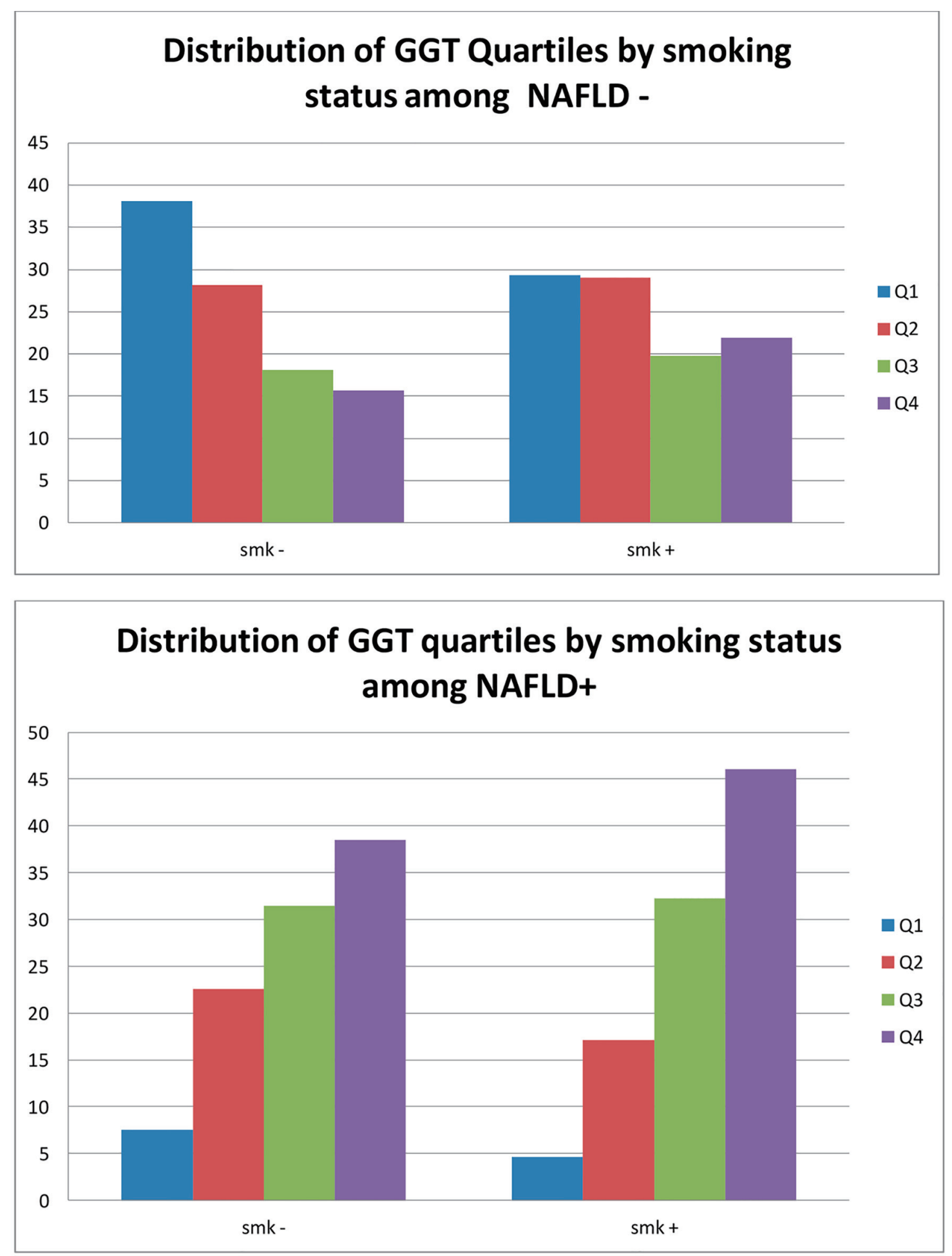

Figure 2. Distribution of GGT quartiles by smoking status and presence of NAFLD. GGT: gamma-glutamyl transferase; NAFLD: non-alcoholic fatty liver disease.

sulin resistance (IR) [35, 37, 38], which may play a role in the development/progression of HS, a component of NAFLD [24]. Importantly, it has been demonstrated that smoking is a risk factor for glucose intolerance and diabetes [39-41]. NAFLD pathogenesis has been linked to IR and the promotion of steatogenesis by increasing the availability of free fatty acids and glucose from adipose tissue and excess dietary intake. This increases de novo hepatic lipogenesis, decrease fatty acid oxi- 

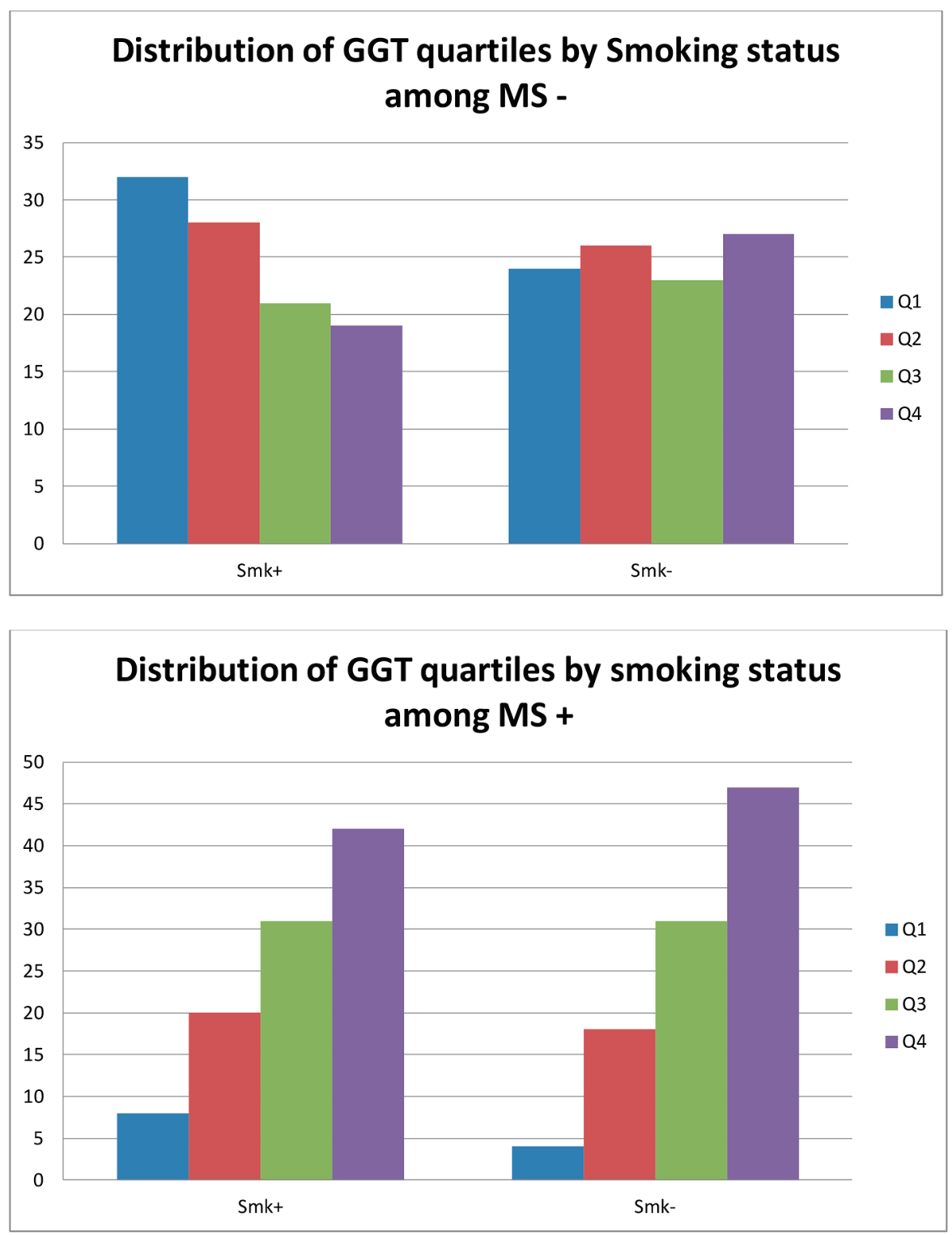

Figure 3. Distribution of GGT quartiles by smoking status and presence of metabolic syndrome. GGT: gamma-glutamyl transferase.

dation and decrease release of lipids from hepatocytes into circulation $[18,42]$. Hamabe et al reported cigarette smoking was an independent risk factor for the onset of HS [24]. This study was supported by Lui et al, who reported that passive smoking and heavy active smoking were both associated with prevalent HS [36]. In a cross-sectional study subjects with NAFLD who smoked had a higher frequency of the MS compared to subjects with NAFLD who did not smoke [43]. Experimental data have also reported that smoking could worsen liver injury in NAFLD [44]. Smoking encourages the production of activated NADPH oxidase-induced reactive species, which enhances oxidative stress and lipid peroxidation [24]. Oxidative stress is a known mechanism of injury in HS $[35,45]$. This study shows that smoking increased GGT levels in general and much more among persons with HS. As expected a similar relationship was seen with MS and obesity. Our finding supports previous studies that have shown that smokers had higher GGT levels [46-48].

GGT is an enzyme responsible for the extracellular catabolism of the antioxidant glutathione [49, 50]. Although used as a diagnostic marker for hepato-bilary disease and alcohol abuse, strong epidemiological and clinical evidence suggests that serum GGT is a potential biomarker for CV morbidity and mortality [51]. Population studies reported significant independent associations between serum GGT concentrations and risk factors, like hypertension, type 2 diabetes and stroke independent of alcohol consumption [6, 8, 10, 15, 52]. Although the mechanism of this association is not clearly defined, it is likely related to oxidative stress $[49,53]$, which could be due to the GGT-linked pro-oxidant effect of glutathione catabolism 
Table 2. Linear Regression of the Association Between Smoking and GGT Level Stratified by NAFLD

\begin{tabular}{lllll}
\hline & $\begin{array}{l}\text { Total (standard- } \\
\text { ized coefficient) }\end{array}$ & HS+ (standardized coefficient) & $\begin{array}{l}\text { HS- (standard- } \\
\text { ized coefficient) }\end{array}$ & $\begin{array}{l}\text { Interaction (HS- } \\
\text { smoking) }\end{array}$ \\
\hline Model 1 & $6.83(3.69-9.96)^{* * *}$ & $10.31(4.68-15.95)^{* * *}$ & $2.36(-1.14-5.85)$ & $\mathrm{P}=0.012$ \\
Model 2 & $6.51(3.46-9.56)^{* * *}$ & $10.19(4.57-15.81)^{* * *}$ & $2.57(-0.83-5.9)$ & $\mathrm{P}=0.015$ \\
Model 3 & $4.65(1.61-7.69)^{* *}$ & $11.12(5.76-16.48)^{* * *}$ & $-0.02(-3.59-3.56)$ & $\mathrm{P}<0.001$ \\
\hline
\end{tabular}

${ }^{*} \mathrm{P}<0.05,{ }^{* *} \mathrm{P}<0.005,{ }^{* * *} \mathrm{P}<0.001$. Model 1: unadjusted. Model 2: adjusted for age, gender. Model 3: model $2+\mathrm{SBP}$, waist circumference, fasting glucose, lipid lowering medications, hypertension meds, LDL-C, AST, alcohol consumption and C-reactive protein. GGT: gamma-glutamyl transferase; NAFLD: non-alcoholic fatty liver disease; HS: hepatic steatosis; SBP: systolic blood pressure; LDL-C: low-density lipoprotein cholesterol; AST: aspartate aminotransferase.

Table 3. Linear Regression of the Association Between Smoking and GGT Level Stratified by Obesity

\begin{tabular}{llll}
\hline & Obesity $+($ standardized coefficient) & Obesity- (standardized coefficient) & Interaction (obesity-smoking) \\
\hline Model 1 & $11.14(3.24-19.03)^{*}$ & $4.65(1.43-7.87)^{*}$ & $\mathrm{P}=0.072$ \\
Model 2 & $10.46(2.64-18.27)^{*}$ & $4.60(1.48-7.73)^{* *}$ & $\mathrm{P}=0.096$ \\
Model 3 & $12.73(5.5-19.96)^{* *}$ & $1.74(-1.45-4.94)$ & $\mathrm{P}<0.001$ \\
\hline
\end{tabular}

${ }^{*} \mathrm{P}<0.05,{ }^{* *} \mathrm{P}<0.005,{ }^{* *} \mathrm{P}<0.001$. Model 1: unadjusted. Model 2: adjusted for age, gender. Model 3: model $2+$ SBP, fasting glucose, lipid lowering medications, hypertension meds, LDL-C, AST, alcohol consumption and C-reactive protein. GGT: gamma-glutamyl transferase; SBP: systolic blood pressure; LDL-C: low-density lipoprotein cholesterol; AST: aspartate aminotransferase.

[49]. GGT notably participates in the proliferation of atheromatous plaques [50] and it is believed that oxidative stress may be the mediating factor of the association between GGT levels and CVD through atherosclerosis [49, 53]. GGT plays a key role in maintaining intracellular anti-oxidant systems through its mediation of extracellular glutathione transport into cells. The association of elevated GGT with CVD is remarkable; Wannamethee et al described a significant relationship

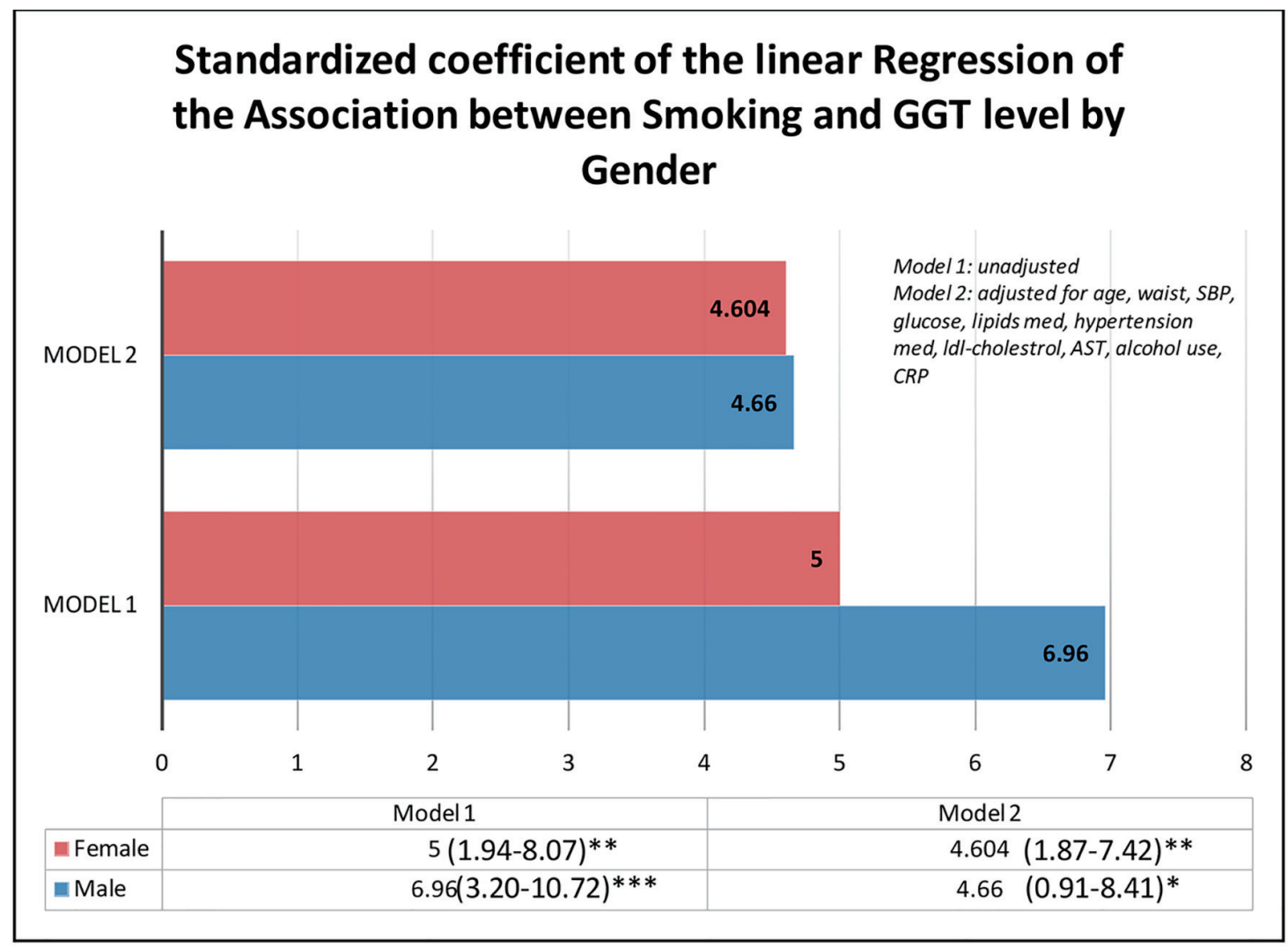

Figure 4. $\beta$-coefficient of the linear regression of smoking and GGT level stratified by gender. GGT: gamma-glutamyl transferase. 
between GGT and CV events in a large prospective study with follow-up up to 24 years [54]. Furthermore, increased GGT levels have been demonstrated to be connected to coronary artery stenoses [1,9]. GGT forms complexes with lipoprotein within atheromatous lesions and in plaques [9, 50].

However, there are questions whether the $\mathrm{CV}$ risk of increased GGT is modifiable [1]. Although there is no convincing evidence that risk for $\mathrm{CV}$ events is lessened by decreasing GGT, there are existing reports of the use of fibrates reducing GGT concentrations in individuals with hypertriglyceridemia, improving their lipid profile $[55,56]$. This may be related to the role of fatty liver in generating increased serum GGT and its association with an increased CV risk [1, 57]. Since NAFLD is mostly treated by life style modifications, serum GGT levels could also be modifiable through these measures.

\section{Implications}

Our study provides evidence that GGT, an increasingly recognized biomarker for CV risk, may be worsened by cigarette smoking in persons with non-alcoholic liver fat. This further re-enforces the importance of life style modifications among persons with NAFLD. Although the presence of NAFLD is an established risk for future CV morbidity and mortality, smoking provides additional insult to the liver and ultimately aggravates the risk. Since oxidative stress has been implicated in many disease processes, including inflammation and atherosclerosis, this study provides further motivation for physicians to emphasize both screening for smoking and smoking cessation efforts in the routine care of individuals with HS and MS. Individuals with elevated GGT may be candidates for NAFLD screening.

\section{Study limitations}

There are a few limitations in this study. First, our study is cross-sectional and as such inferences are weakened as temporality cannot be ascertained. Secondly, the classification of smoking status as current smoking and non-smoker did not provide any information on history of smoking and as such presents the challenge of a residual bias. Also, there was no information on the quantity of cigarettes smoked, as such a dose response association could not be verified. Thirdly, the number of smokers with HS compared to the rest of the population may be a limitation to the interpretation of the results. Fourthly, our diagnosis of liver fat was based on ultrasound findings. Although ultrasound is a useful non-invasive and cheap tool for identifying NAFLD, its sensitivity for detecting fatty changes within the liver is reduced when less than moderate severity $[28,58]$. Imaging using ultrasound is most effective when greater than a third of the liver is affected.

\section{Strengths}

Despite the limitations, this study has relevant strengths. An important strength for this study is the large number of par- ticipants free of coronary heart disease. This has allowed for adjustment for multiple confounding risk factors to ascertain the relationship. The cohort size also allowed for evaluation of the relationship within demographic and clinical subgroups.

\section{Conclusion}

Oxidative stress plays a significant step in the development and progression of CVD. Serum GGT is an established and measurable biomarker of oxidative stress. In this study of asymptomatic individuals, smoking significantly raised oxidative stress as measured by GGT level among persons with NAFLD. While elevated GGT levels are associated with both NAFLD and CVD risk, smoking may further worsen this risk. Although future longitudinal studies to ascertain this association are imperative, this study provides further motivation for physicians to emphasize smoking screening and cessation in the routine care of individuals with NAFLD and MS.

\section{Acknowledgments}

We would like to acknowledge the efforts of MB, RS, RC and $\mathrm{JC}$ in acquiring the data used for this study.

\section{Financial Disclosure}

None to declare.

\section{Conflict of Interest}

None to declare.

\section{Informed Consent}

This study did not involve any animal study and appropriate IRB approval was obtained prior to the administration of surveys to the participants.

\section{Author Contributions}

$\mathrm{EO}, \mathrm{EA}, \mathrm{MB}, \mathrm{EV}$ and $\mathrm{KN}$ contributed to the conception, analysis and interpretation of the data. EO and $\mathrm{KN}$ performed the statistical analysis. EO, VF, MB, RC, JC, JM, RS and KN were involved in drafting the manuscript and revising it critically for important intellectual content. All authors read and approved the final manuscript.

\section{Abbreviations}

ALT: alanine aminotransferase; AST: aspartate aminotransferase; AUDIT: alcohol use disorders identification test; CVD: 
cardiovascular disease; CV: cardiovascular; DM: diabetes mellitus; GGT: gamma-glutamyl transferase; HDL-C: highdensity lipoprotein cholesterol; hs-CRP: high-sensitivity Creactive protein; HS: hepatic steatosis; IR: insulin resistance; NADPH: nicotinamide adenine dinucleotide phosphate (reduced); NAFLD: non-alcoholic fatty liver disease; PHI: protected health information

\section{References}

1. Turgut O, Yilmaz A, Yalta K, Karadas F, Birhan Yilmaz M. gamma-Glutamyltransferase is a promising biomarker for cardiovascular risk. Med Hypotheses. 2006;67(5):10601064.

2. Pleiner J, Mittermayer F, Schaller G, Marsik C, MacAllister RJ, Wolzt M. Inflammation-induced vasoconstrictor hyporeactivity is caused by oxidative stress. J Am Coll Cardiol. 2003;42(9):1656-1662.

3. Violi F, Pignatelli P. Clinical Application of NOX Activity and Other Oxidative Biomarkers in Cardiovascular Disease: A Critical Review. Antioxid Redox Signal. 2015;23(5):514-532.

4. Whitfield JB. Gamma glutamyl transferase. Crit Rev Clin Lab Sci. 2001;38(4):263-355.

5. Glass GA, Stark AA. Promotion of glutathione-gammaglutamyl transpeptidase-dependent lipid peroxidation by copper and ceruloplasmin: the requirement for iron and the effects of antioxidants and antioxidant enzymes. Environ Mol Mutagen. 1997;29(1):73-80.

6. Lee DS, Evans JC, Robins SJ, Wilson PW, Albano I, Fox CS, Wang TJ, et al. Gamma glutamyl transferase and metabolic syndrome, cardiovascular disease, and mortality risk: the Framingham Heart Study. Arterioscler Thromb Vasc Biol. 2007;27(1):127-133.

7. Expert Panel on Detection Evaluation, Treatment of High Blood Cholesterol in Adults. Executive summary of the third report of the National Cholesterol Education Program (NCEP) expert panel on detection, evaluation, and treatment of high blood cholesterol in adults (Adult Treatment Panel III). JAMA. 2001;285(19):2486-2497.

8. Wannamethee G, Ebrahim S, Shaper AG. Gammaglutamyltransferase: determinants and association with mortality from ischemic heart disease and all causes. Am J Epidemiol. 1995;142(7):699-708.

9. Emdin M, Passino C, Michelassi C, Titta F, L'Abbate A, Donato L, Pompella A, et al. Prognostic value of serum gamma-glutamyl transferase activity after myocardial infarction. Eur Heart J. 2001;22(19):1802-1807.

10. Ruttmann E, Brant LJ, Concin H, Diem G, Rapp K, U1mer H, Vorarlberg Health M, et al. Gamma-glutamyltransferase as a risk factor for cardiovascular disease mortality: an epidemiological investigation in a cohort of 163,944 Austrian adults. Circulation. 2005;112(14):21302137.

11. Hozawa A, Okamura T, Kadowaki T, Murakami Y, Nakamura K, Hayakawa T, Kita Y, et al. gamma-Glutamyltransferase predicts cardiovascular death among Japanese women. Atherosclerosis. 2007;194(2):498-504.
12. Emdin M, Passino C, Michelassi C, Donato L, Pompella A, Paolicchi A. Additive prognostic value of gamma-glutamyltransferase in coronary artery disease. Int J Cardiol. 2009;136(1):80-85.

13. Kawamoto R, Kohara K, Tabara Y, Miki T, Otsuka N. Serum gamma-glutamyl transferase levels are associated with metabolic syndrome in community-dwelling individuals. J Atheroscler Thromb. 2009;16(4):355-362.

14. Loomba R, Doycheva I, Bettencourt R, Cohen B, Wassel CL, Brenner D, Barrett-Connor E. Serum gammaglutamyltranspeptidase predicts all-cause, cardiovascular and liver mortality in older adults. J Clin Exp Hepatol. 2013;3(1):4-11.

15. Meisinger C, Doring A, Schneider A, Lowel H, the Group KS. Serum gamma-glutamyltransferase is a predictor of incident coronary events in apparently healthy men from the general population. Atherosclerosis. 2006;189(2):297302.

16. Ishizaka N, Ishizaka Y, Toda E, Yamakado M, Koike K, Nagai R. Association between gamma-glutamyltransferase levels and insulin resistance according to alcohol consumption and number of cigarettes smoked. J Atheroscler Thromb. 2010;17(5):476-485.

17. Hu G, Tuomilehto J, Pukkala E, Hakulinen T, Antikainen R, Vartiainen E, Jousilahti P. Joint effects of coffee consumption and serum gamma-glutamyltransferase on the risk of liver cancer. Hepatology. 2008;48(1):129-136.

18. Bhatia LS, Curzen NP, Byrne CD. Nonalcoholic fatty liver disease and vascular risk. Curr Opin Cardiol. 2012;27(4):420-428.

19. Fraser A, Harris R, Sattar N, Ebrahim S, Smith GD, Lawlor DA. Gamma-glutamyltransferase is associated with incident vascular events independently of alcohol intake: analysis of the British Women's Heart and Health Study and Meta-Analysis. Arterioscler Thromb Vasc Biol. 2007;27(12):2729-2735.

20. Heitzer T, Yla-Herttuala S, Luoma J, Kurz S, Munzel T, Just H, Olschewski M, et al. Cigarette smoking potentiates endothelial dysfunction of forearm resistance vessels in patients with hypercholesterolemia. Role of oxidized LDL. Circulation. 1996;93(7):1346-1353.

21. Sackett DL, Gibson RW, Bross ID, Pickren JW. Relation between aortic atherosclerosis and the use of cigarettes and alcohol. An autopsy study. N Engl J Med. 1968;279(26):1413-1420.

22. Jonas MA, Oates JA, Ockene JK, Hennekens CH. Statement on smoking and cardiovascular disease for health care professionals. American Heart Association. Circulation. 1992;86(5):1664-1669.

23. Kannel WB. Update on the role of cigarette smoking in coronary artery disease. Am Heart J. 1981;101(3):319328.

24. Hamabe A, Uto H, Imamura Y, Kusano K, Mawatari S, Kumagai K, Kure T, et al. Impact of cigarette smoking on onset of nonalcoholic fatty liver disease over a 10-year period. J Gastroenterol. 2011;46(6):769-778.

25. Agarwal R. Smoking, oxidative stress and inflammation: impact on resting energy expenditure in diabetic nephropathy. BMC Nephrol. 2005;6:13. 
26. Avti PK, Kumar S, Pathak CM, Vaiphei K, Khanduja KL. Smokeless tobacco impairs the antioxidant defense in liver, lung, and kidney of rats. Toxicol Sci. 2006;89(2):547553.

27. Muriel P. Role of free radicals in liver diseases. Hepatol Int. 2009;3(4):526-536.

28. Ndumele CE, Nasir K, Conceicao RD, Carvalho JA, Blumenthal RS, Santos RD. Hepatic steatosis, obesity, and the metabolic syndrome are independently and additively associated with increased systemic inflammation. Arterioscler Thromb Vasc Biol. 2011;31(8):1927-1932.

29. Perloff D, Grim C, Flack J, Frohlich ED, Hill M, McDonald M, Morgenstern BZ. Human blood pressure determination by sphygmomanometry. Circulation. 1993;88(5 Pt 1):2460-2470.

30. Bellentani S, Saccoccio G, Masutti F, Croce LS, Brandi G, Sasso F, Cristanini G, et al. Prevalence of and risk factors for hepatic steatosis in Northern Italy. Ann Intern Med. 2000;132(2):112-117.

31. Lazo M, Hernaez R, Bonekamp S, Kamel IR, Brancati FL, Guallar E, Clark JM. Non-alcoholic fatty liver disease and mortality among US adults: prospective cohort study. BMJ. 2011;343:d6891.

32. Grundy SM, Cleeman JI, Daniels SR, Donato KA, Eckel RH, Franklin BA, Gordon DJ, et al. Diagnosis and management of the metabolic syndrome: an American Heart Association/National Heart, Lung, and Blood Institute Scientific Statement. Circulation. 2005;112(17):27352752.

33. Barbor TF, Higgins-Biddle J, Saunders JB, Monteiro MG. The alcohol use disorders identification test: guidelines for use in primary care (vol. 2). World Health Organization. 2001.

34. Willenbring ML, Massey SH, Gardner MB. Helping patients who drink too much: an evidence-based guide for primary care clinicians. Am Fam Physician. 2009;80(1):44-50.

35. Zein CO, Unalp A, Colvin R, Liu YC, McCullough AJ, Nonalcoholic Steatohepatitis Clinical Research N. Smoking and severity of hepatic fibrosis in nonalcoholic fatty liver disease. J Hepatol. 2011;54(4):753-759.

36. Liu Y, Dai M, Bi Y, Xu M, Xu Y, Li M, Wang T, et al. Active smoking, passive smoking, and risk of nonalcoholic fatty liver disease (NAFLD): a population-based study in China. J Epidemiol. 2013;23(2):115-121.

37. Attvall S, Fowelin J, Lager I, Von Schenck H, Smith U. Smoking induces insulin resistance - a potential link with the insulin resistance syndrome. J Intern Med. 1993;233(4):327-332.

38. Janzon L, Berntorp K, Hanson M, Lindell SE, Trell E. Glucose tolerance and smoking: a population study of oral and intravenous glucose tolerance tests in middleaged men. Diabetologia. 1983;25(2):86-88.

39. Wannamethee SG, Shaper AG, Perry IJ, British Regional Heart S. Smoking as a modifiable risk factor for type 2 diabetes in middle-aged men. Diabetes Care. 2001;24(9):1590-1595.

40. Houston TK, Person SD, Pletcher MJ, Liu K, Iribarren C, Kiefe CI. Active and passive smoking and development of glucose intolerance among young adults in a prospective cohort: CARDIA study. BMJ. 2006;332(7549):10641069.

41. Will JC, Galuska DA, Ford ES, Mokdad A, Calle EE. Cigarette smoking and diabetes mellitus: evidence of a positive association from a large prospective cohort study. Int J Epidemiol. 2001;30(3):540-546.

42. Kopec KL, Burns D. Nonalcoholic fatty liver disease: a review of the spectrum of disease, diagnosis, and therapy. Nutr Clin Pract. 2011;26(5):565-576.

43. Chiang PH, Chang TY, Chen JD. Synergistic effect of fatty liver and smoking on metabolic syndrome. World J Gastroenterol. 2009;15(42):5334-5339.

44. Yuan H, Shyy JY, Martins-Green M. Second-hand smoke stimulates lipid accumulation in the liver by modulating AMPK and SREBP-1. J Hepatol. 2009;51(3):535-547.

45. Sanyal AJ, Campbell-Sargent C, Mirshahi F, Rizzo WB, Contos MJ, Sterling RK, Luketic VA, et al. Nonalcoholic steatohepatitis: association of insulin resistance and mitochondrial abnormalities. Gastroenterology. 2001;120(5):1183-1192.

46. Steffensen FH, Sorensen HT, Brock A, Vilstrup H, Lauritzen T. Alcohol consumption and serum liver-derived enzymes in a Danish population aged 30-50 years. Int J Epidemiol. 1997;26(1):92-99.

47. Jang ES, Jeong SH, Hwang SH, Kim HY, Ahn SY, Lee $\mathrm{J}$, Lee $\mathrm{SH}$, et al. Effects of coffee, smoking, and alcohol on liver function tests: a comprehensive cross-sectional study. BMC Gastroenterol. 2012;12:145.

48. Lee DH, Lim JS, Yang JH, Ha MH, Jacobs DR, Jr. Serum gamma-glutamyltransferase within its normal range predicts a chronic elevation of alanine aminotransferase: a four year follow-up study. Free Radic Res. 2005;39(6):589-593.

49. Emdin M, Pompella A, Paolicchi A. Gamma-glutamyltransferase, atherosclerosis, and cardiovascular disease: triggering oxidative stress within the plaque. Circulation. 2005;112(14):2078-2080.

50. Paolicchi A, Emdin M, Passino C, Lorenzini E, Titta F, Marchi S, Malvaldi G, et al. Beta-lipoprotein- and LDL-associated serum gamma-glutamyltransferase in patients with coronary atherosclerosis. Atherosclerosis. 2006;186(1):80-85.

51. Turgut O, Yilmaz MB, Yalta K, Tandogan I. Gamma-glutamyltransferase as a useful predictor for cardiovascular risk: clinical and epidemiological perspectives. Atherosclerosis. 2009;202(2):348-349.

52. Perry IJ, Wannamethee SG, Shaper AG. Prospective study of serum gamma-glutamyltransferase and risk of NIDDM. Diabetes Care. 1998;21(5):732-737.

53. Schulze PC, Lee RT. Oxidative stress and atherosclerosis. Curr Atheroscler Rep. 2005;7(3):242-248.

54. Wannamethee SG, Lennon L, Shaper AG. The value of gamma-glutamyltransferase in cardiovascular risk prediction in men without diagnosed cardiovascular disease or diabetes. Atherosclerosis. 2008;201(1):168-175.

55. Fukuo Y, Kitami T, Nomoto T, Terashi A. A lipid lowering drug (bezafibrate) has a favorable effect on liver enzymes (Al-P and gamma-GTP). Nihon Ika Daigaku Zasshi. 
1996;63(5):424-430.

56. Mikhailidis DP, Ganotakis ES, Spyropoulos KA, Jagroop IA, Byrne DJ, Winder AF. Prothrombotic and lipoprotein variables in patients attending a cardiovascular risk management clinic: response to ciprofibrate or lifestyle advice. Int Angiol. 1998;17(4):225-233.

57. Targher G, Bertolini L, Poli F, Rodella S, Scala L, Tessari
$\mathrm{R}$, Zenari L, et al. Nonalcoholic fatty liver disease and risk of future cardiovascular events among type 2 diabetic patients. Diabetes. 2005;54(12):3541-3546.

58. Saadeh S, Younossi ZM, Remer EM, Gramlich T, Ong JP, Hurley M, Mullen KD, et al. The utility of radiological imaging in nonalcoholic fatty liver disease. Gastroenterology. 2002;123(3):745-750. 